\title{
Salivary epidermal growth factor correlates with hospitalization length in rotavirus infection
}

\author{
J. Gómez-Rial ${ }^{1,2 *}$, M. J. Curras-Tuala ${ }^{1}$, C. Talavero-González ${ }^{1,2}$, C. Rodríguez-Tenreiro ${ }^{1}$, L. Vilanova-Trillo', \\ A. Gómez-Carballa ${ }^{1,4,5}$, I. Rivero-Calle ${ }^{1,3}$, A. Justicia-Grande ${ }^{1,3}$, J. Pardo-Seco ${ }^{1,4,5}$, L. Redondo-Collazo 1,3, \\ A. Salas ${ }^{1,4,5}$ and F. Martinón-Torres ${ }^{1,3}$
}

\begin{abstract}
Background: The IFI27 interferon gene expression has been found to be largely increased in rotavirus (RV)-infected patients. IF/27 gene encodes for a protein of unknown function, very recently linked to epidermal proliferation and related to the epidermal growth factor (EGF) protein. The EGF is a low-molecular-weight polypeptide that is mainly produced by submandibular and parotid glands, and it plays an important physiological role in the maintenance of oro-esophageal and gastric tissue integrity.

Our aim was to determine salivary EGF levels in RV-infected patients in order to establish its potential relationship with IFI27 increased expression and EGF-mediated mucosal protection in RV infection.
\end{abstract}

Methods: We conducted a prospective comparative study using saliva samples from 27 infants infected with RV (sampled at recruitment during hospital admission and at convalescence, i.e. at least 3 months after recovery) and from 36 healthy control children.

Results: Median (SD) EGF salivary concentration was 777 (529) pg/ml in RV-infected group at acute phase and 356 (242) $\mathrm{pg} / \mathrm{m}$ at convalescence, while it was 337 (119) $\mathrm{pg} / \mathrm{ml}$ in the healthy control group. A significant association was found between EGF levels and hospitalization length of stay ( $P$-value $=0.022 ; r^{2}=-0.63$ ).

Conclusions: The salivary levels of EGF are significantly increased during the acute phase of natural RV infection, and relate to length of hospitalization. Further assessment of this non-invasive biomarker in RV disease is warranted.

Keywords: Biomarkers, EGF, IFI27, Rotavirus infection

\section{Background}

Rotavirus (RV) infection is the most common cause of severe diarrhoea in children and may cause serious dehydration that usually requires hospitalization [1]. Predominantly, but not exclusively, RV infects the terminally differentiated enterocytes in the small intestine and it induces mucosal damage, villus atrophy and necrosis of epithelial cells [2]. RV infection alters the function of the

\footnotetext{
*Correspondence: jose.gomez.rial@sergas.es

'Grupo de Investigación en Genética, Vacunas, Infecciones y Pediatría (GENVIP), Hospital Clínico Universitario de Santiago de Compostela, Santiago de Compostela, Galicia, Spain

2Laboratorio de Inmunología, Servicio de Análisis Clínicos, Hospital

Clínico Universitario de Santiago de Compostela, Santiago de

Compostela, Galicia, Spain

Full list of author information is available at the end of the article
}

small intestinal epithelium, resulting in characteristic diarrhoea, secondary to enterocyte destruction.

There are currently not reliable specific biomarkers for RV infection. Identifying new correlates of disease activity may help in designing new treatments and more efficacious RV vaccines. Use of saliva as a proxy indicator of intestinal immunity has been proposed as a non-invasive source of biomarkers [3]. Salivary IgA has been recently suggested as a correlate for protection for norovirus intestinal infection [4].

In a recent whole blood transcriptomic analysis of RVinfected patients, we reported a 23.7 fold increase in the expression of the IFI27 gene, an interferon-related gene with unknown function in the RV infection [5]. Moreover, this gene has recently been associated to the 
epidermal growth factor (EGF) and involved in proliferation of epithelia [6]. EGF, also known as epithelial growth factor, is a single chain polypeptide secreted by the submandibular salivary glands and other exocrine intestinal glands. In vivo actions of EGF in the gastrointestinal tract include promoting wound healing and tissue repair, inducing epithelium proliferation and promoting differentiation [7]. A significant contribution of salivary EGF to the maintenance of the integrity of the oesophageal mucosa has been demonstrated in experimental and clinical settings [8]. Patients with low salivary EGF levels are predisposed to severe oesophageal damage by gastro-oesophageal reflux [9].

The aim of the present study was to measure the levels of EGF in saliva from acute-phase RV-infected children and to assess its potential relationship with disease course and severity.

\section{Methods}

\section{Patients and controls}

This is a prospective single-centre case-control study consisting of the collection of saliva samples and clinical data from pediatric patients admitted to hospital due to a RV infection, and age- and gender-matched healthy controls. A total of 27 patients with RV infection were prospectively recruited at the Pediatric Department of Hospital Clínico Universitario of Santiago de Compostela (Spain) during the period 2013-2014, all of them hospitalized with acute gastroenteritis and with a positive RV antigen detected in stool. Demographic and clinical data, including fever, vomiting, diarrhoea, dehydration and total severity scores during entire period of illness were obtained. During the same period, 36 healthy controls were enrolled in the study.

Saliva samples were collected at recruitment on admission to the hospital (acute phase) and at convalescence (at least 90 days after infection) for the RV-infected group. A single sample was obtained at recruitment for healthy controls group.

The saliva sample was obtained from unstimulated sublingual saliva using oral swabs (Whatman; Maidstone, Kent, UK) placed under the tongue for $5 \mathrm{~min}$. The swabs were eluted in $0.4 \mathrm{ml}$ of phosphate buffered saline (PBS), and then centrifuged at $800 \mathrm{~g}$ for $10 \mathrm{~min}$ to remove mucin and epithelial cells. Supernatants were stored at $-30{ }^{\circ} \mathrm{C}$ prior to analysis.

\section{Clinical data collection}

Temperature, number of vomiting episodes per day and duration of vomiting, the severity of diarrhoea (numbers of stools per day, duration of diarrhoea, and level of dehydration), length of hospital stay, and total symptoms scores measured using Vesikari's score were registered in RV-infected subjects.
Table 1 Demographic characteristics of the study patients expressed as mean (SD)

\begin{tabular}{llll}
\hline Variable & $\begin{array}{l}\text { RV } \\
\text { acute-phase }\end{array}$ & $\begin{array}{l}\text { RV } \\
\text { convalescence }\end{array}$ & $\begin{array}{l}\text { Healthy } \\
\text { controls }\end{array}$ \\
\hline Demographic data & & & 36 \\
$n$ & 27 & 23 & $7(6-7)$ \\
Age [months (SD)] & $12.5(1-40)$ & $18(5-47)$ & $18: 18$ \\
Sex (male:female) & $16: 11$ & $14: 9$ & \\
\hline
\end{tabular}

\section{Detection of EGF in saliva samples}

EGF detection assay was carried out using Milliplex Map human cytokine detection kit (Merck Millipore, Billerica, MA), following manufacturer indications. Assays were carried out on a Luminex ${ }^{\text {tx }} 200$ platform.

\section{Statistical analysis}

Data are expressed as median and interquartile range. Statistical analyses were performed using the statistical software R v.3.1.1 (http://www.r-project.org). Outliers were identified as those values falling above or below boxplot whiskers, and eliminated from further analysis. Non-parametric statistics were used for analysis because data were not normally distributed in the cohort of cases. Wilcoxon rank-sum tests were used for comparison between acute-phase versus convalescence samples and versus healthy controls. Spearman's rank correlation coefficients were used to quantify the association between EGF concentration and disease clinical parameters. Reported $P$-values were below the Bonferroni significant threshold.

\section{Results}

Patients in the RV-infected group ranged in age from 1 to 40 months (median of 12.5 months) at recruitment. Healthy control children ranged in age from 6 to 7 months (median of 7 months). Demographic and clinical characteristics are summarized in Tables 1 and 2.

Median levels for EGF in saliva from acute-phase RVinfected patients were $777(\mathrm{SD}=529) \mathrm{pg} / \mathrm{ml}$. Salivary EGF

Table 2 Clinical characteristics of patients in acute phase expressed as mean (SD)

\begin{tabular}{ll}
\hline Clinical data & RV acute-phase \\
\hline Vesikari's score & $11.07(3.485)$ \\
Length of stay in hospital (days) & $5.750(3.026)$ \\
Temperature $\left({ }^{\circ} \mathrm{C}\right)$ & $38.70(0.622)$ \\
Number of vomiting episodes per day & $3.179(4.738)$ \\
Duration of vomiting (days) & $2.037(1.931)$ \\
Number of stools per day & $4.929(5.018)$ \\
Duration of diarrhoea (days) & $4.571(2.168)$ \\
Level of dehydration & $1.607(1.100)$ \\
\hline
\end{tabular}



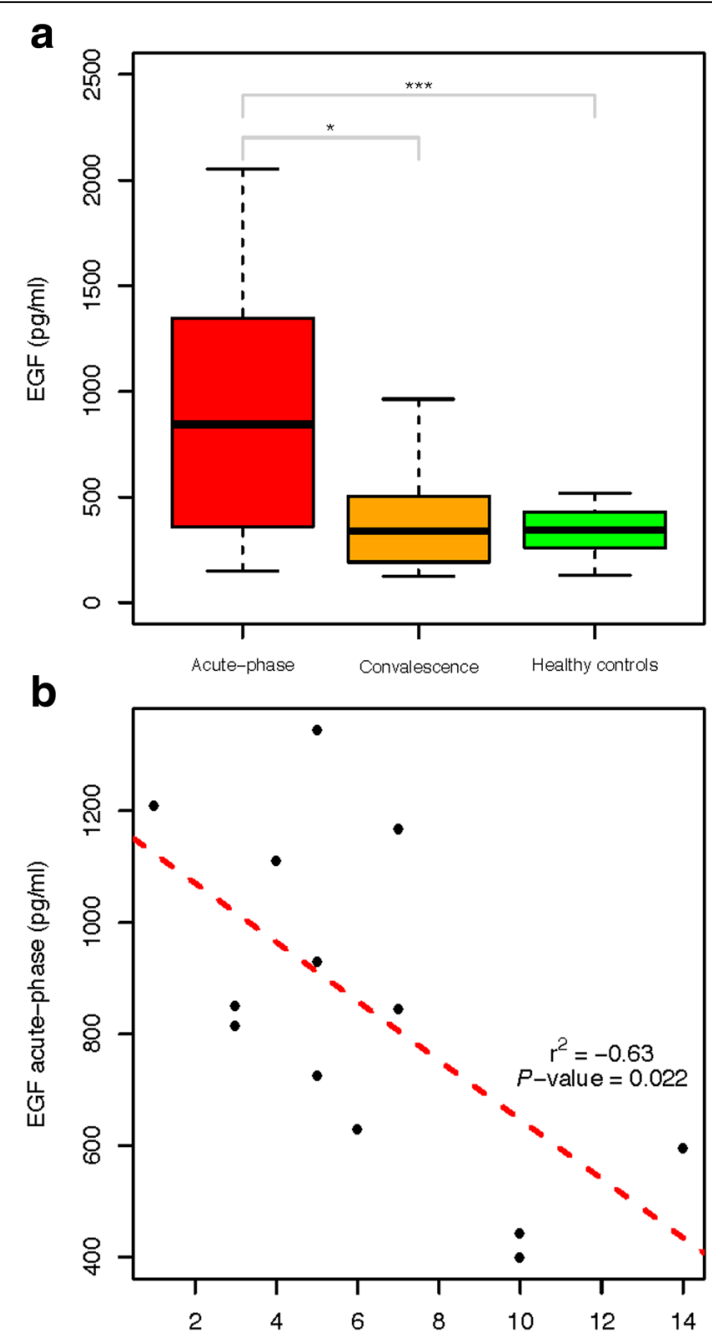

C

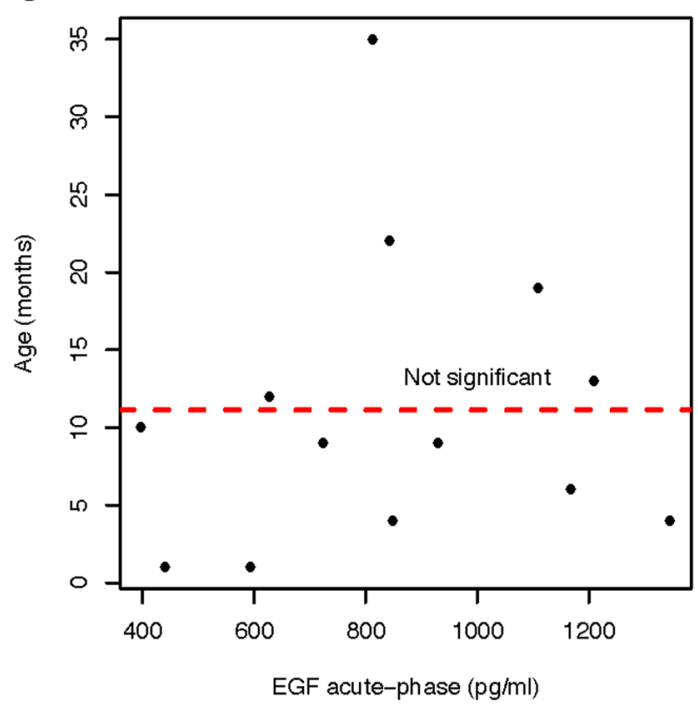

Fig. 1 a Values of EGF measured in saliva from RV-infected patients during acute phase $(n=27)$ and convalescence $(n=23)$, and in healthy controls $(n=36)$. Saliva samples were tested for EGF using multiplex cytokine analysis as described in the text. Results are presented as median and interquartile range EGF levels in pg/ml. A non-parametric Wilcoxon test was used to determine statistical significance between acute-phase and convalescence samples. Mann-Whitney tests were used for comparison between acutephase and healthy controls. $P$-values indicated on graphs are $<0.05$ $\left(^{*}\right)$ and $<0.0001\left(^{* * *}\right)$. b Association between salivary EGF level and length of hospitalization stay. Salivary EGF levels and symptom scores for 27 RV-infected patients were analysed as described in the text. No other significant association was detected. Spearman's rank correlation coefficient $\left(r^{2}\right)$ is presented $(P$-value $=0.022)$. $\mathbf{c}$ Age and salivary EGF levels. No association was observed between EGF level and age

levels where significantly lower in convalescent patients $(356 \mathrm{pg} / \mathrm{ml} ; \mathrm{SD}=242$; Wilconxon test, $P$-value $=0.015)$ and healthy controls $(337 \mathrm{pg} / \mathrm{ml} ; \mathrm{SD}=119$; Wilconxon test, $P$-value $=0.001474)$; Fig. $1 \mathrm{a}$. These variations in EGF levels were not age-related (Fig. 1b).

When assessing possible correlations between EGF levels and clinical variables in RV infected subjects, a significant negative correlation was found between salivary EGF concentration and length of hospital stay $\left(P\right.$-value $\left.=0.022 ; r^{2}=-0.63\right)$. We did not find any other significant association between EGF levels and other clinical parameters of disease (Fig. 1c).

\section{Discussion}

In a previous whole blood transcriptomic analysis [5] carried out in RV-infected patients vs control children, a remarkable over-expression of IFI27 gene was reported. IFI27 belongs to the family of interferon inducible genes, and recently it has been associated to epidermal proliferation and EGF [6]. In addition, IFI27 was previously identified as a marker of epithelial cancers and psoriatic lesions [10] and related to systemic lupus erythematosus (SLE) [11]. However, the exact role of IFI27 in the RV infection physiopathology is still unknown, although based on our results we can suggest that RV infection might activate an interferon-mediated mechanism of mucosal recovery after infection damage through a pathway mediated by IFI27. The observed association between IFI27 expression and EGF requires a more indepth analysis, and the mechanistic approach needs to be clarified.

EGF is linked to epithelia regeneration after mucosal lesions, promoting mucosal wound healing and tissue repair [12]. Previous studies in neonatal pigs infected with RV already pointed to a beneficial role of high physiological levels of EGF in stimulating recovery of epithelium in the small intestine following infection [13]. EGF application in the mucosa can promote intestinal proliferation and improve restoration after intestinal injury, 
and it might be an effective treatment against intestinal ischemia-reperfusion injury in rats [14]. The hypothesis of EGF supplementation after intestinal injury as a potential treatment for improving outcome in patients has been put forward by other authors based on animal studies [15]. This finding might lay the ground for a new recovery treatment after RV infection.

Our present study shows that salivary levels of EGF are increased during natural RV infection and that they may correlate with the severity of the disease in terms of length of hospital stay. The over-expression of EGF levels in infected patients might suggest a host recovery response to the damage induced by RV in the intestinal mucosa. The disruption in the normal homeostasis of mucosa would induce the production of EGF, also expressed in the submandibular salivary glands, in order to restore the integrity of mucosa after infection.

Interestingly, we found that the higher the levels of EGF in acute-phase samples from RV-infected patients upon admission, the shorter the length of stay in hospital. It could be argued that the greater level of EGF reflects a more powerful response of mucosal homeostasis, thus facilitating a faster recovery, and a reduction in the length of hospitalization. This association, if prospectively confirmed, might allow to make predictions on recovery of mucosal integrity and hospitalization length with a simple and non-invasive methodology. The use of saliva as a non-invasive proxy for intestinal immunity after enteric infection opens the door to the search for new specific biomarkers for RV infection that might correlate with clinical parameters of disease.

One limitation of the present study is the limited number of individual analysed, however the novelty of our findings merits consideration and further confirmatory studies. Another limitation derives from the different localization of previous gene expression analyses (performed in whole blood), and our EGF quantification (performed in the epithelial mucosa).

\section{Conclusions}

In conclusion, RV infection induces an increased production of EGF by submandibular glands that could be associated to IFI27 gene over-expression. Moreover, increased salivary EGF concentrations were found to be associated with length of hospitalization. Further studies to corroborate our findings are needed. In the meantime, we can postulate saliva as a good proxy to advance in our knowledge of RV infection.

\section{Abbreviations}

EGF: Epidermal growth factor; IFI27: Interferon-alpha inducible protein 27; RV: Rotavirus; SLE: Systemic Lupus Erythematous

Acknowledgement

Not applicable.

\section{Funding}

This study received support from the Instituto de Salud Carlos III (Proyecto de Investigación en Salud, Acción Estratégica en Salud): project GePEM ISCIII/ PI16/01478/Cofinanciado FEDER) (A.S.) and project Rotanext ISCIII/PI13/02382 and ReSVinext ISCIII/PI16/01569/Cofinanciado FEDER (F.M.T.); Consellería de Sanidade, Xunta de Galicia (RHI07/2-intensificación actividad investigadora, PS09749 and 10PXIB918184PR), Instituto de Salud Carlos III (Intensificación de la actividad investigadora 2007-2012, PI16/01569), Fondo de Investigación Sanitaria (FIS; PI070069/PI1000540) del plan nacional de I + D + I and 'fondos FEDER' (F.M.T.), and 2016-PG071 Consolidación e Estructuración REDES 2016GI-1344 G3VIP (Grupo Gallego de Genética Vacunas Infecciones y Pediatría, ED341D R2016/021)(A.S. and F.M.T) Funding sources have had no involvement in the design of the study, collection, analysis and interpretation of data and in writing the manuscript.

\section{Availability of data and materials}

The datasets used and/or analyzed during the current study are available from the corresponding author on reasonable request.

\section{Authors' contributions}

JGR conceived the study, participated in its design and wrote the manuscript. MJCT and CTG carried out the immunoassays. CRT participated in the design of the study and ethical statement. LVT, IRC, LRC and AJG participated in the recruitment of patients. AGC participated in processing of samples. JPS and AS performed the statistical analysis. AS and FMT participated in study design and co-ordination, and contributed to draft the manuscript. All authors read and approved the final manuscript.

Competing interests

The authors declare that they have no competing interests.

\section{Consent for publication}

Not applicable.

\section{Ethics approval and consent to participate}

The study was approved by the reference independent ethics committee of Galicia-SERGAS and conducted in compliance with the Declaration of Helsinki, the International Conference on Harmonisation, Good Clinical Practice Guidelines, and all local regulatory requirements. Written informed consent was obtained from all parents or legal guardians before enrolment and before undertaking any study-related procedures.

\section{Publisher's Note}

Springer Nature remains neutral with regard to jurisdictional claims in published maps and institutional affiliations.

\section{Author details}

${ }^{1}$ Grupo de Investigación en Genética, Vacunas, Infecciones y Pediatría (GENVIP), Hospital Clínico Universitario de Santiago de Compostela, Santiago de Compostela, Galicia, Spain. ${ }^{2}$ Laboratorio de Inmunología, Servicio de Análisis Clínicos, Hospital Clínico Universitario de Santiago de Compostela, Santiago de Compostela, Galicia, Spain. ${ }^{3}$ Translational Pediatrics and Infectious Diseases, Department of Pediatrics, Hospital Clínico Universitario de Santiago de Compostela, Santiago de Compostela, Galicia, Spain. ${ }^{4}$ Unidade de Xenética, Departamento de Anatomía Patolóxica e Ciencias Forenses, Instituto de Ciencias Forenses, Facultade de Medicina, Universidade de Santiago de Compostela, Santiago de Compostela, Galicia, Spain. ${ }^{5}$ GenPob Research Group, Instituto de Investigaciones Sanitarias (IDIS), Hospital Clínico Universitario de Santiago, Santiago de Compostela, Galicia, Spain.

Received: 28 October 2016 Accepted: 15 May 2017

Published online: 30 May 2017

\section{References}

1. Fischer TK, Viboud C, Parashar U, Malek M, Steiner C, Glass R, et al. Hospitalizations and deaths from diarrhea and rotavirus among children $<5$ years of age in the United States, 1993-2003. J Infect Dis. 2007;195(8):1117-25.

2. Rivero-Calle I, Gomez-Rial J, Martinon-Torres F. Systemic features of rotavirus infection. J Inf Secur. 2016;72:S98-S105. 
3. Aase A, Sommerfelt $H$, Petersen LB, Bolstad M, Cox RJ, Langeland N, et al. Salivary IgA from the sublingual compartment as a novel noninvasive proxy for intestinal immune induction. Mucosal Immunol. 2015;9(4):884-93.

4. Ramani S, Neill FH, Opekun AR, Gilger MA, Graham DY, Estes MK, et al. Mucosal and cellular immune responses to Norwalk virus. J. Infect. Dis. 2015; 212(3):397-405.

5. Salas A, Marco-Puche G, Triviño JC, Gomez CA, Cebey-López M, Rivero-Calle I, et al. Strong down-regulation of glycophorin genes: a host defense mechanism against rotavirus infection. Infect Genet Evol. 2016;44:403-11.

6. Hsieh WL, Huang YH, Wang TM, Ming YC, Tsai CN, Pang JH. IFI27, a novel epidermal growth factor-stabilized protein, is functionally involved in proliferation and cell cycling of human epidermal keratinocytes. Cell Prolif. 2015;48(2):187-97.

7. Walters JR. Cell and molecular biology of the small intestine: new insights into differentiation, growth and repair. Curr Opin Gastroenterol. 2004;20(2):70-6.

8. Suzuki A, Sekiya S, Gunshima E, Fujii S, Taniguchi H. EGF signaling activates proliferation and blocks apoptosis of mouse and human intestinal stem/ progenitor cells in long-term monolayer cell culture. Lab Investig. 2010; 90(10):1425-36.

9. Eckley CA, Sardinha LR, Rizzo LV. Salivary concentration of epidermal growth factor in adults with reflux laryngitis before and after treatment. Ann Otol Rhinol Laryngol. 2013;122(7):440-4.

10. Suomela S, Cao L, Bowcock A, Saarialho-Kere U. Interferon alpha-inducible protein 27 (IFI27) is upregulated in psoriatic skin and certain epithelial cancers. J Invest Dermatol. 2004;122(3):717-21.

11. Kyogoku C, Smiljanovic B, Grun JR, Biesen R, Schulte-Wrede U, Haupl T, et al. Cell-specific type I IFN signatures in autoimmunity and viral infection: what makes the difference? PLoS One. 2013;8(12):e83776.

12. Tarnawski AS, Jones MK. The role of epidermal growth factor (EGF) and its receptor in mucosal protection, adaptation to injury, and ulcer healing: involvement of EGF-R signal transduction pathways. J Clin Gastroenterol. 1998:27(Suppl 1):S12-20.

13. Zijlstra RT, Odle J, Hall WF, Petschow BW, Gelberg HB, Litov RE. Effect of orally administered epidermal growth factor on intestinal recovery of neonatal pigs infected with rotavirus. J Pediatr Gastroenterol Nutr. 1994; 19(4):382-90.

14. Geng Y, Li J, Wang F, Li Q, Wang X, Sun L, et al. Epidermal growth factor promotes proliferation and improves restoration after intestinal ischemiareperfusion injury in rats. Inflammation. 2013;36(3):670-9.

15. Jung K, Kang BK, Kim JY, Shin KS, Lee CS, Song DS. Effects of epidermal growth factor on atrophic enteritis in piglets induced by experimental porcine epidemic diarrhoea virus. Vet J. 2008;177(2):231-5.

\section{Submit your next manuscript to BioMed Central and we will help you at every step:}

- We accept pre-submission inquiries

- Our selector tool helps you to find the most relevant journal

- We provide round the clock customer support

- Convenient online submission

- Thorough peer review

- Inclusion in PubMed and all major indexing services

- Maximum visibility for your research

Submit your manuscript at www.biomedcentral.com/submit 